\title{
Motion Capture Based Model Identification of the Humanoid Robot REEM-C Using Static Poses
}

\author{
$\underline{\text { Felix Aller }}^{1}$, Monika Harant ${ }^{2}$ and Katja Mombaur ${ }^{3}$ \\ ${ }^{1}$ Institute of Computer Engineering \\ Heidelberg University \\ Berliner Str. 45, 69120 Heidelberg, Germany \\ felix.aller@ziti.uni-heidelberg.de \\ ${ }^{2}$ Department of Mathematics for the Digital Factory \\ Fraunhofer Institute for Industrial Mathematics \\ Fraunhofer-Platz 1, 67663 Kaiserslautern, Germany \\ monika.harant@itwm.fraunhofer.de \\ ${ }^{3}$ CERC Human-Centred Robotics and Machine Intelligence \\ University of Waterloo \\ 200 University Avenue West, Waterloo, Canada, N2L 3G1 \\ katja.mombaur@uwaterloo.ca
}

\begin{abstract}
In this paper, we describe an approach for the model identification of the humanoid robot REEM-C. In contrast to previous work, we do not attempt to determine all dynamic parameters simultaneously. It is not clear whether such approaches can lead to redundancies in the optimization problem. We deliberately restrict ourselves to a very precise determination of the center-of-mass (COM) and the mass of the individual rigid bodies. As a result, we do not use Persistent Exciting (PE) trajectories and perform the identification based on motion capture and force plate measurements of 172 static poses. This results in more accurate experimental data and allows a more precise update of static parameters by means of an optimization problem. The inertial parameters are not updated and have to be adjusted using classical approaches, but based on the already improved static parameters. We report the performance of optimization by comparing the distance of the ground-projected-center-of-mass (GCOM) against the measured GCOM from the model information of the original and optimized model for each static pose. The improvement of the optimized model is furthermore reflected by means of a recorded dynamic squat motion and by analyzing the residual torques and forces acting at the floating base of the robot.
\end{abstract}

Keywords: multibody dynamics, humanoid robot, inertial parameters, model identification.

\section{INTRODUCTION}

Humanoid robots are on the advance with capabilities that make them suitable for use outside of laboratory environments. Their application in collaboration with humans or for tasks that are too dangerous or monotonous for humans is thus within reach $[1,2,3]$. Due to their anthropomorphic structure, they have advantages in a complex working environment made by humans for humans [4] and their bipedal locomotion makes it possible to master more challenging terrain [5]. This advantage comes at a cost, as an upright posture and locomotion is not statically stable for most configurations, as is the case for multi-legged robots. Thus, many motion sequences push the robot to its limits in order to perform complex tasks while maintaining static and dynamic stability [6].

There are many different approaches to generating motion sequences for humanoids, and those that reach a robot's limits exploit the full dynamic properties described in the robot's model [7]. 
For the challenging task of developing whole-body motions on complex multibody systems such as humanoid robots, knowledge of the exact dynamic properties of the mechanical system are required. This is further complicated by inaccuracies in the dynamic model parameters for which we can distinguish between four different categories:

1. The geometry or kinematic structure of the robot, which is usually generated from the underlying CAD files and is very precise.

2. The mass and centers-of-masses of all rigid bodies involved, which is also exported from $\mathrm{CAD}$, but often only reflects a fraction of the individual (electrical) components within the rigid body.

3. The moments of inertia, which are also derived from the CAD. A uniform density distribution within the individual rigid bodies is often assumed. For this reason, the moments of inertia are also only an approximation of the body with individual components of different unknown densities.

4. Limitations of the robot in terms of joint angles, torques or velocity. These can be determined fairly accurately based on the motor specification.

As category 2 and 3 are mostly mere, flawed approximations of the actual parameters, much attention is paid to model identification using multibody dynamics, initially for manipulators in the 1980 's $[8,9,10]$. This method was extended to be applicable to humans [11] and humanoid robots $[12,13]$. Subsequent work further extends this method by also including joint torque information of the actuated joints [14]. Each method requires a set of trajectories, also called Persistent Exciting (PE) trajectories with the aim to excite all the dynamics of the system $[15,16]$. The two methods differ in the way that the former requires the torques to be calculated from external contact forces using inverse dynamics, whereas the latter method obtains the torques directly from the robot's force torque sensors in each joint. Both methods have already been compared by applying them to a state-of-the-art humanoid [17].

A general disadvantage of dynamic model identification based on PE-trajectories lies in the fact that acceleration data is approximated, which can lead to errors in the parameter estimation. Furthermore, most robots, such as the one used in this work, are not equipped with sensors to measure joint torques leading to errors due to the estimation of torque from contact forces. It has also not yet been investigated to what extent the simultaneous optimization of all dynamic parameters can lead to redundancies in the optimization problem.

In this work, we carry out a model identification procedure on the humanoid robot REEM-C by PAL Robotics for parameters of category 2 . We focus on identifying and update center of mass locations and masses so that the model best fits the experimental data, which consists of a large set of static poses. The approach of splitting the simultaneous identification of static and dynamic parameters of categories 2 . and 3. respectively and the use of static poses allows a more accurate approximation of 2. to reality. This approximation can subsequently serve as a basis for the more precise identification of the dynamic parameters. The level of model improvement is evaluated by means of a dynamic reference motion.

This paper is organized as follows. In Section 2, we describe the humanoid robot REEM-C and the kinematic and dynamic modeling of an anthropomorphic system. In Section 3, we present the carried out method for model identification. The results of the updated parameter sets are described in Section 4. The results are discussed in Section 5. Section 6 ends the paper with a conclusion and perspectives for future research. 

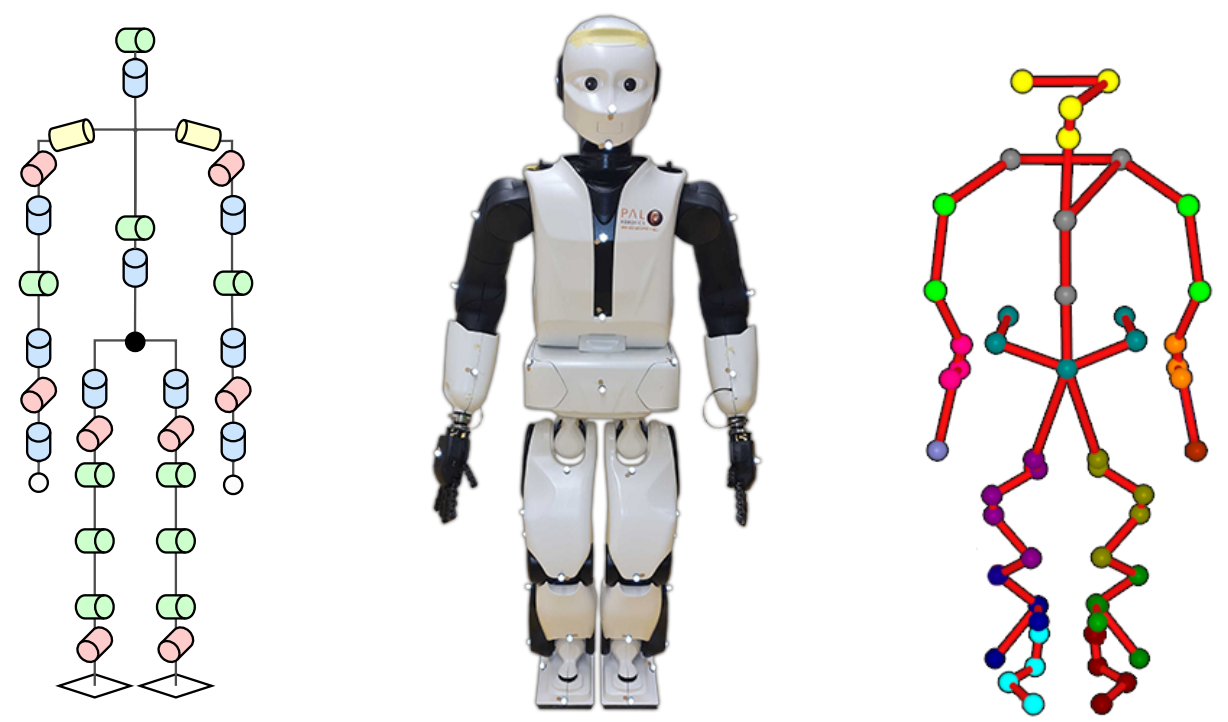

Figure 1. Kinematic structure of REEM-C with 30 (actual) DOF without hands (2 head, 7 each arm, 2 waist, 6 each leg, 19 each hand) [18], the real robot with applied marker set [18], and the actual marker set consisting of 51 optical markers.

\section{ANTHROPOMORPHIC DESCRIPTION}

\subsection{Humanoid robot REEM-C}

REEM-C is a humanoid robot developed by PAL Robotics. With its $1.64 \mathrm{~m}$ size the robot is considered an adult sized robot (Fig. 1 center). It features 30 DOF (Fig. 1 left) not considering each hand with 19 additional DOF. This is roughly the same amount as the number of DOF of comparable robots such as ARMAR-4 (41), WALK-MAN (31), HRP-2 (30), Talos (30) or DRC-HUBO+ (28) again, not considering the varying DOF of each of the robots' hands. All joints of REEM-C are revolute and powered by high gain driving brushed DC motors except for the underactuated hands. Each motor encoder can track the position and velocity of the motion characteristic, as external perturbations are rejected by the position controlled motors. REEM-C is equipped with multiple sensors such as an IR-camera, force torque sensors in the ankles and wrists and an IMU located in the waist, which is also hosting the batteries of the robot and is the heaviest rigid body in the system. The robotic feet are rubber coated with a sole size of $21.0 \mathrm{~cm} \times 14.0 \mathrm{~cm}$. However, the actual support polygon of REEM-C while standing on one leg is estimated to be $15.5 \mathrm{~cm} \times$ $10.6 \mathrm{~cm}$, which is a significantly smaller area. The total weight of the robot was measured and amounts to $77.6 \mathrm{~kg}$, whereas the robotic model only accounts for $67.55 \mathrm{~kg}$.

Even if this weight difference seems large, it can easily be justified. PAL Robotics is always adapting newer versions of the robot to different requirements. In this regard, REEM-C has been equipped with more powerful knee motors in the current version 4 . In addition, the classic generation of the initial model from the available CAD files does not take electrical wiring into account. Furthermore, a homogeneous battery and a homogeneous computer are assumed. The paint and the lubricant of the motors are not considered. There are also different manufacturing tolerances and the same material is not always used for the robot's casing, although the different materials used are considered to be identical.

The joint configuration of REEM-C does not contain any closed loop and is therefore modeled using a kinematic tree structure. The origin of the whole system is called the floating base, the freeflyer or the base link. The floating base determines the global position and orientation of the robot with respect to the inertial frame. This state is achieved by adding an additional 6-DOF joint 
connecting the floating base to the inertial frame. This results in a system consisting of

$$
q=\left[\begin{array}{ll}
q_{b} & q_{j}
\end{array}\right]^{T},
$$

where

$$
q_{J}=\left[\begin{array}{lll}
q_{0} & \cdots & q_{n}
\end{array}\right]^{T} .
$$

The number of actual joints is represented by $n \in \mathbb{N}$. The configuration of the virtual joints to achieve the global state in terms of translation and rotation of the floating base is indicated by $q_{b} \in \mathbb{R}^{6}$. The joint configurations of the actuated joints is represented by $q_{j} \in \mathbb{R}^{n}$. This gives a total number of DOF of $n+6$ where only $n$ joints are actually actuated so the resulting system is always underactuated, regardless of the size of $n$. We therefore obtain a maximum number of 74 DOF for REEM-C.

\subsection{Dynamic Modeling}

The development of dynamic models is an important part of the implementation of motion trajectories for robots. Especially in model-based trajectory generation, the quality of the resulting motion depends on the individual model parameters. Target trajectories generated on the basis of models naturally vary in complexity. Accordingly, simpler motion sequences such as the typical zero-moment-point based walking of a humanoid are more forgiving of model inaccuracies. With more complex motion sequences, model inaccuracies become increasingly severe, such as when crossing obstacles [19]. If additional velocity requirements are added to a challenging trajectory for highly dynamic and impulsive motion sequences, even the smallest model inaccuracies make execution on a real robot impossible. The latter category includes movements that shift the pelvis of the multibody system system along the vertical axis, such as sit-to-stand transitions. Therefore, the description of the robotic system must correspond as closely as possible to reality.

A rigid body system can be described using the equation of motion which can be written in the following form as a set of ODEs:

$$
H(q, p) \ddot{q}+N(q, \dot{q}, p)+C(q, \dot{q}, p)=F(q, \dot{q}, p, \tau)
$$

where $q, \dot{q}$ and $\ddot{q}$ are vectors of the joint coordinates of position, velocity and acceleration, respectively. The generalized inertia matrix which is dependent on $q$ and $p$ is written as $H(q, p)$. Likewise, the acting gravity which depends on $q$ and $\dot{q}$ is denoted as $C(q, \dot{q})$. The produced torque by the actuation system is written as $F(q, \dot{q}, p, \tau)$, which also includes external forces.

The generalized inertia matrix of the robot and its corresponding motors can be further described as

$$
H(q, p)=\sum_{b=1}^{n}\left(J_{b}^{x}\right)^{T} m_{b} J_{b}^{x}+\left(J_{b}^{\omega}\right)^{T} I_{b} J_{b}^{\omega} .
$$

The nonlinear effects $N(q, \dot{q}, p)$ are described as:

$$
\left.N(q, \dot{q}, p)=\sum_{b=1}^{n}\left(J_{b}^{x}\right)^{T} m_{b} \dot{J}_{b}^{x}+\left(J_{b}^{\omega}\right)^{T} I_{b} \dot{J}_{b}^{\omega}-\left(\left(J_{b}^{\omega}\right)^{T} I_{b} J_{b}^{\omega} \dot{q}\right)\right) J_{b}^{\omega}
$$

where $J_{b}^{x}$ and $J_{b}^{\omega}$ are the Jacobians containing the translatory and rotatory information of each rigid body $b$. The inertia tensor of $b$ in the global frame is denoted as $I_{b}$.

The parameter set which contains the model parameters from categories $1-4$ (see Section 1) is denoted as $p$. As mentioned earlier we focus on the identification of the static parameters $p_{s} \subset p$ : the rigid body masses $m_{b}$ and the center-of-mass (COM) locations of $b$ with respect to the local 
body frame, $c_{b} \in \mathbb{R}^{3}$. Using $m_{b}$ and $c_{b}$ we can describe the mean location of a distribution of mass in space, i.e. the distribution which has the property that the weighted position vectors relative to this point are zero and denote it as $r(q)=(x, y, z)$ with

$$
r(q)=\frac{1}{M} \sum_{b=1}^{n} m_{b} r_{b}\left(q, p_{s}\right),
$$

where $M$ is the total mass of the system. The global position of $c_{b}$ is obtained by $r_{b}$ based on the current configuration $q$ of the robot. The ground-projected-center-of-mass (GCOM) is defined as $G:=\left(x, y, h_{G}\right)$, with $h_{G}$ being the floor height.

\section{APPLIED METHOD FOR MODEL IDENTIFICATION}

While previous work has attempted to determine all dynamic parameters simultaneously, our decision to focus on $p_{s}$ allows a identification based on static poses. Therefore no PE trajectories are needed. This benefits the data quality and accuracy of model improvement. Additionally, we do not rely on the internal sensor values of the robot, as we aim for the highest possible precision of the measured values. In the case of REEM-C, the motor encoders do not take into account gear backlash and an overall mechanical system tilt was observed when the GCOM reached the edge of the support polygon. Therefore, we utilize measurement systems from a state-of-the-art motion capture lab. This method is particularly suitable for robots that do not have the necessary sensors or measuring accuracy to determine the external contact forces and position information of the rigid bodies. This makes this method completely robot-independent and applicable to all kinds of different multi-body systems. Subsequently, the inertia matrices are to be estimated, but on the basis of the optimized, already determined static parameters.

\subsection{Static poses}

Static poses offer the advantage of taking a measurement at a stage when the robot is almost at rest and the measurements are nearly free of noise. One disadvantage, however, is that a large amount of poses are required. A combination of systematic and randomly generated poses was used. We distinguish 6 categories of poses (Fig. 2 left to right):

1. Double-legged - feet $2 \mathrm{~cm}$ apart on one force plate, focus on right arm,

2. Single-legged - standing on the left leg, focus on right leg,

3. Single-legged - standing on the right leg, focus on left leg,

4. Double-legged - feet $2 \mathrm{~cm}$ apart on one force plate, focus on torso joints (Fig. 3(a)),

5. Double-legged - feet $2 \mathrm{~cm}$ apart on one force plate, crouching,

6. Double-legged - feet $20 \mathrm{~cm}$ apart with both feet on one force plate (Fig. 3(b)) and both feet on one force plate each (Fig. 3(c)),

A total of 172 static poses was generated. Each pose is a static joint configuration, i.e. the GCOM resides within the convex hull of the support polygon. For each configuration, the individual degrees of freedom were systematically set to less than $10 \%$ and more than $90 \%$ of their minimum and maximum joint range. In addition, at least 10 random poses were generated for the doublelegged categories.

The kinematic data were collected using a passive motion capture system at $150 \mathrm{~Hz}$ (Qualisys, Gothenburg, Sweden) and two ground-embedded force plates at $900 \mathrm{~Hz}$ (Bertec, Columbus, OH, USA). We applied a custom marker set on REEM-C with 51 motion capture markers (14 mm) 


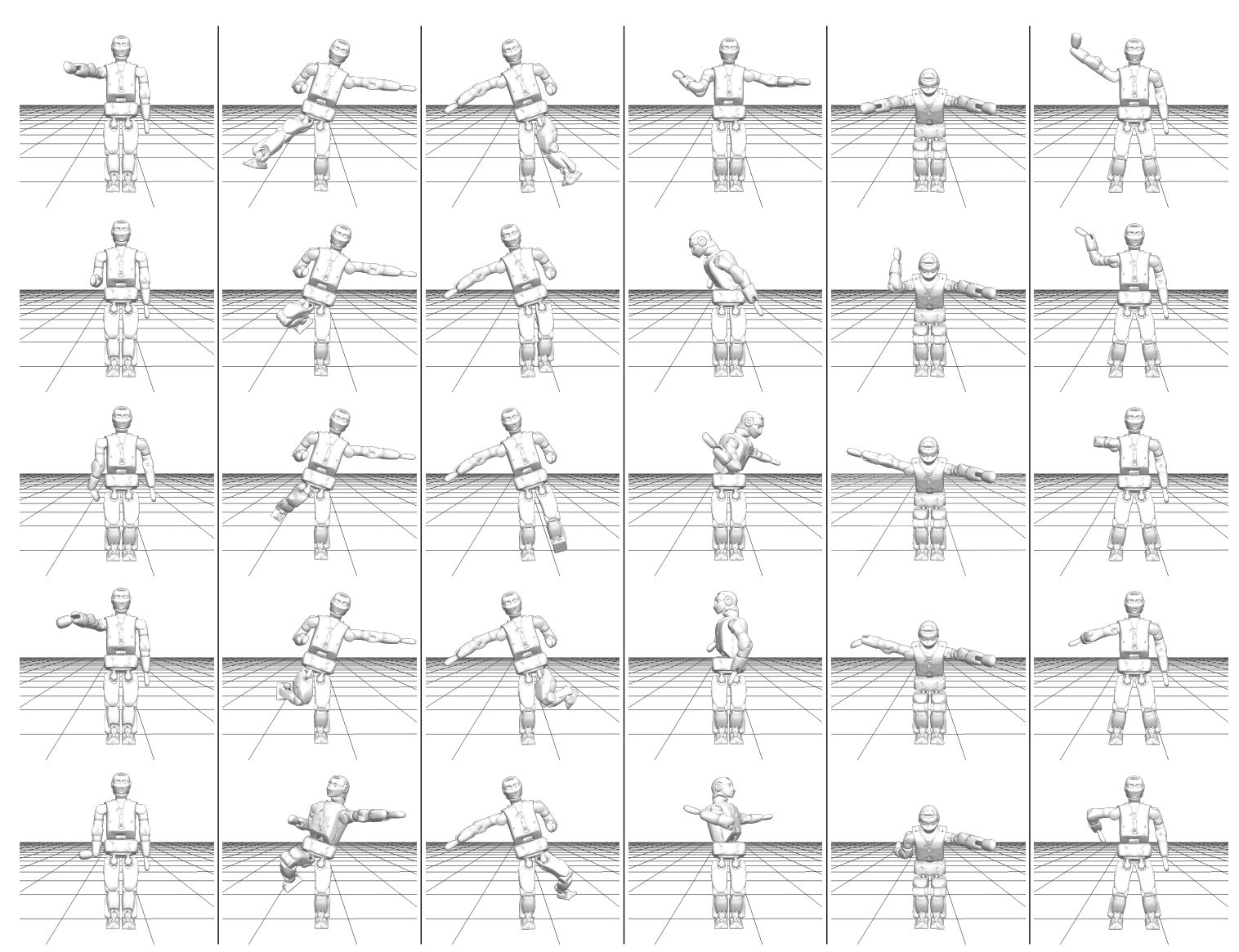

Figure 2. Examples from the applied static poses. From left to right: (1.) On both feet, (2.) On left foot, (3.) On right foot, (4.) Torso rotation, (5.) Crouching, (6.) Both feet (wide).

(Fig. 1 right). The marker and force data were filtered with a bi-directional Butterworth filter with a cut-off frequency of $10 \mathrm{~Hz}$.

For each of the static poses, we use the ground-embedded force plates to measure the corresponding center-of-pressure (COP). The COP is a dynamic point described by the forces exerted by two rigid bodies in contact. Generally, we consider the rigid body of one or more feet of the robotic system and the ground surface on which the robot is standing. Let us consider the simplified case of a humanoid with one foot in contact with the ground. The contact force is always positive because the foot cannot penetrate the ground. The COP describes a point at which the sum of all compressive forces perpendicular to the sole of the foot is equivalent to a single force acting at a point where the resulting moment is exactly zero. We distinguish two cases: the contact of a single foot, where the COP must always lie within the convex hull spanned by the flat contact of the foot with the ground, and the contact of both feet, where the convex hull is spanned by both foot contact surfaces. In the latter case, the COP can lie between both feet and outside the actual contact area of one foot. In static poses, i.e. a situation in which no other forces act apart from the gravitational forces, the COP corresponds to the GCOM.

\subsection{Kinematic Fitting of the Experimental Data}

For the parameter optimization it is vital to be able to calculate the GCOM for different poses and model parameters and compare it to the measured COP. As mentioned earlier, markers were attached to all segments of the robot and recorded as well to be able to obtain the position of the robot as accurate as possible. The joint positions recorded by the robot itself can not measure a global tilt of the system induced by the gear backlash. 

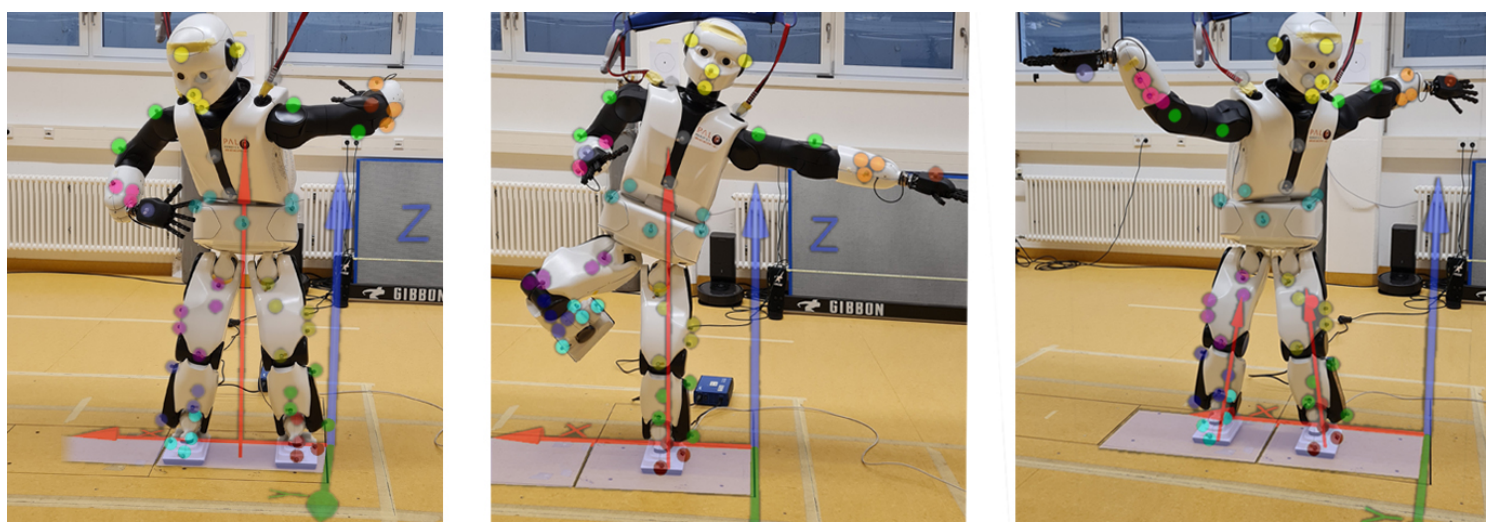

Figure 3. Three different types of static poses of the humanoid REEM-C with applied marker set (highlighted) on force plates (highlighted) with indicated force vector: (a) standing on both feet on one force plate, (b) standing on one foot, (c) standing on both feet on two force plates.

For fitting the marker positions onto the model of REEM-C, virtual markers were included in the model description and their position with respect to the segment they are attached to were determined via measurements as well as a calibration trial with the robot standing in a neutral pose. The joint positions of the trials are obtained by minimizing the distance between virtualmarkers and motion capture markers for each frame:

$$
\min _{q} \sum_{m=1}^{N_{m}}\left\|r_{m}^{V}(q)-r_{m}^{C}\right\|_{2}^{2}
$$

with joint angles $q$, the positions of the virtual markers and of the corresponding motion capture markers are denoted by $r_{m}^{V}$ and $r_{m}^{C}, m=1, \ldots, N_{m}$, respectively, with $N_{m}$ being the total amount of markers used. Note that $q$ are the joint positions of one frame or time instance and also include the position and orientation of the floating base in space.

The optimization problem is solved using the Levenberg-Marquardt method [20] provided by the open source Rigid Body Dynamics Library - RBDL [21]. This iterative method starts at an initial guess and is refining it until a termination criterion is met. It is quite robust with respect to different starting values. However, if the orientation of the model specified by the initial guess is far away from the orientation specified by the motion capture markers, the calculated solution can contain joint angles of over 360 degrees and more, exceeding the range of motion of the robot. In order to avoid this effect, the orientation of the pelvis segment is estimated first using the position of the corresponding motion capture markers, and included in the initial guess of the first frame. The start values of the remaining frames are taken from the previous one.

\subsection{Experimental Parameter Identification}

The recorded marker data are fitted to the model using inverse kinematics. For each static pose, the GCOM obtained by the dynamic properties of the model is compared to the COP obtained through force plate measurements. As described in Section 3.1, the measured COP corresponds to the GCOM during a static pose, and is therefore denoted as GCOM ${ }^{R E F}$. Their deviation is minimized by solving a least squares problem by updating a set of static model parameters $p_{s}$ :

$$
\begin{aligned}
\min _{p_{s}} & \sum_{n=1}^{N}\left\|g_{G C O M}\left(q_{n}, p_{s}\right)-\operatorname{GCOM}_{n}^{R E F}\right\|^{2} \\
\text { s.t. } & g_{\text {mass }}\left(p_{s}\right)=M
\end{aligned}
$$




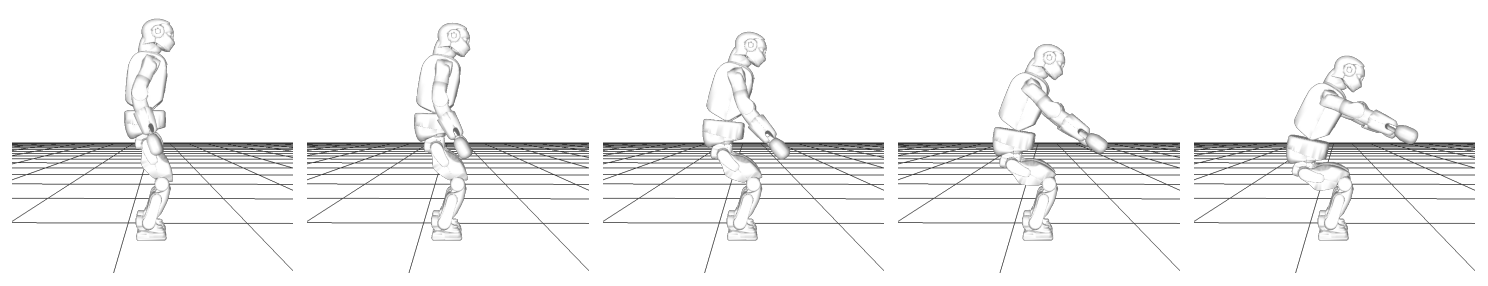

Figure 4. Validation motion for model identification which is comparable to the human squat exercise. The robot shifts the pelvis along the vertical axis and maintains static stability by shifting the arms and upper body within a period of 5 seconds.

with $N$ the number of poses considered and $\operatorname{GCOM}_{m}^{R E F}$ the recorded GCOM of pose $m$. The functions $g_{G C O M}$ and $g_{\text {mass }}$ calculate the GCOM of pose $m$ specified by the recorded joint positions $q_{m}$ and the total mass of the robot model based on the current static parameters $p_{s}$. The measured total mass of the robot is denoted by $M$. The parameters consist of the mass and COM location of the main segments of the robot: shank, thigh, pelvis, trunk, upper arm, forearm and hand. Segments consisting of only a motor and a cover could not be considered during the optimization and were fixed at the values given in the original model, as the change in the measured GCOM would be too small and lead to redundancies in the problem formulation. The same applies to the head and foot segments. The optimization problem is solved with the SQP method using Gauss-Newton Hessian and a Levenberg-Marquardt regularization term using the optimal control software package MUSCOD II [22].

\subsection{Validation of model identification}

For validation of the model identification a reference motion was recorded using the same experimental setup. The chosen motion is similar to the human squat exercise, in which the COM is moved along the vertical plane and at the same time static stability is maintained by shifting the arms and upper body (Fig. 4). The data obtained was not used during the optimization process. The marker data was fitted to the robot model and the torques were calculated using inverse dynamics. To assess the quality of the model, we evaluated the joint torques and forces acting at the floating base. These calculated residual forces should be practically very small compared to the external and internal forces in the equilibrium case for underactuated degrees of freedom. Therefore, they are considered as the actual error of the numerical solution and can be used as a benchmark for the quality of model optimization.

\section{RESULTS}

\subsection{Comparison of static poses}

We evaluate the improved accuracy of the optimization problem by analyzing the distance of the recorded $G C O M^{R E F}$ and the GCOM obtained by the model parameters for both, the original and the optimized model (Fig. 5). The optimization problem was able to reduce the deviations for all 172 poses to varying degrees. An overall distance correction from (avg. $9.2 \mathrm{~mm}$, std. $2.3 \mathrm{~mm}$ ) to (avg. $0.6 \mathrm{~mm}$, std. $0.4 \mathrm{~mm}$ ) is reported. However, the optimization does not decrease the distances to the same extent for all static poses.

\subsection{Residual forces and torques}

The quality of the model identification is evaluated by the analysis of the residual forces and torques against a reference trajectory. We report the residual forces and torques acting at the floating base during the squat motion along all axis and compare the original model against the optimized model after the identification procedure (Fig. 6). For all plots we identify an oscillation 


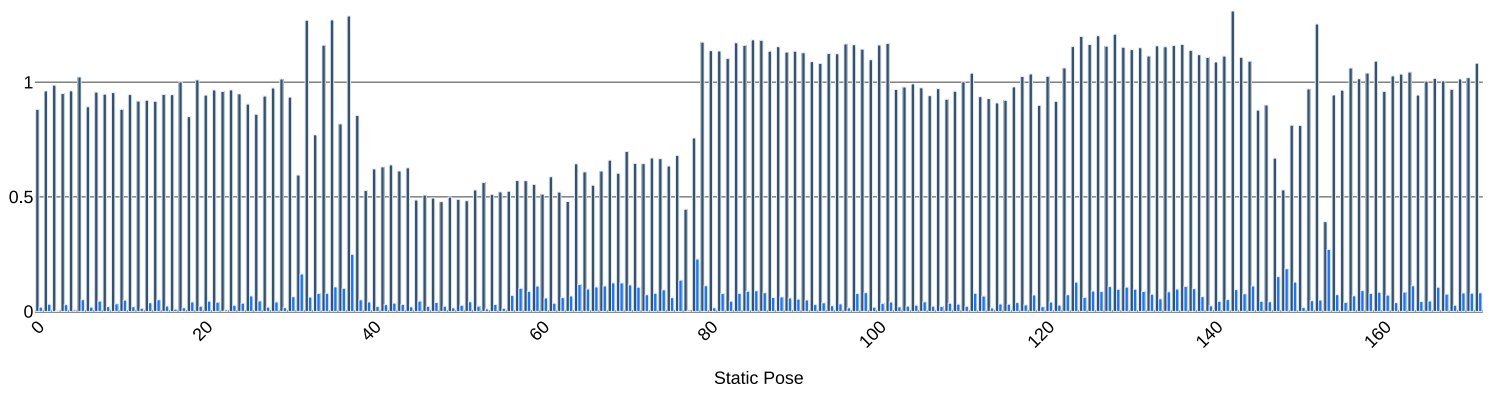

Figure 5. Distance of GCOM to $G C O M^{R E F}$ for all 172 static poses for the original model (dark-blue) and the optimized model (light-blue) obtained by model identification.
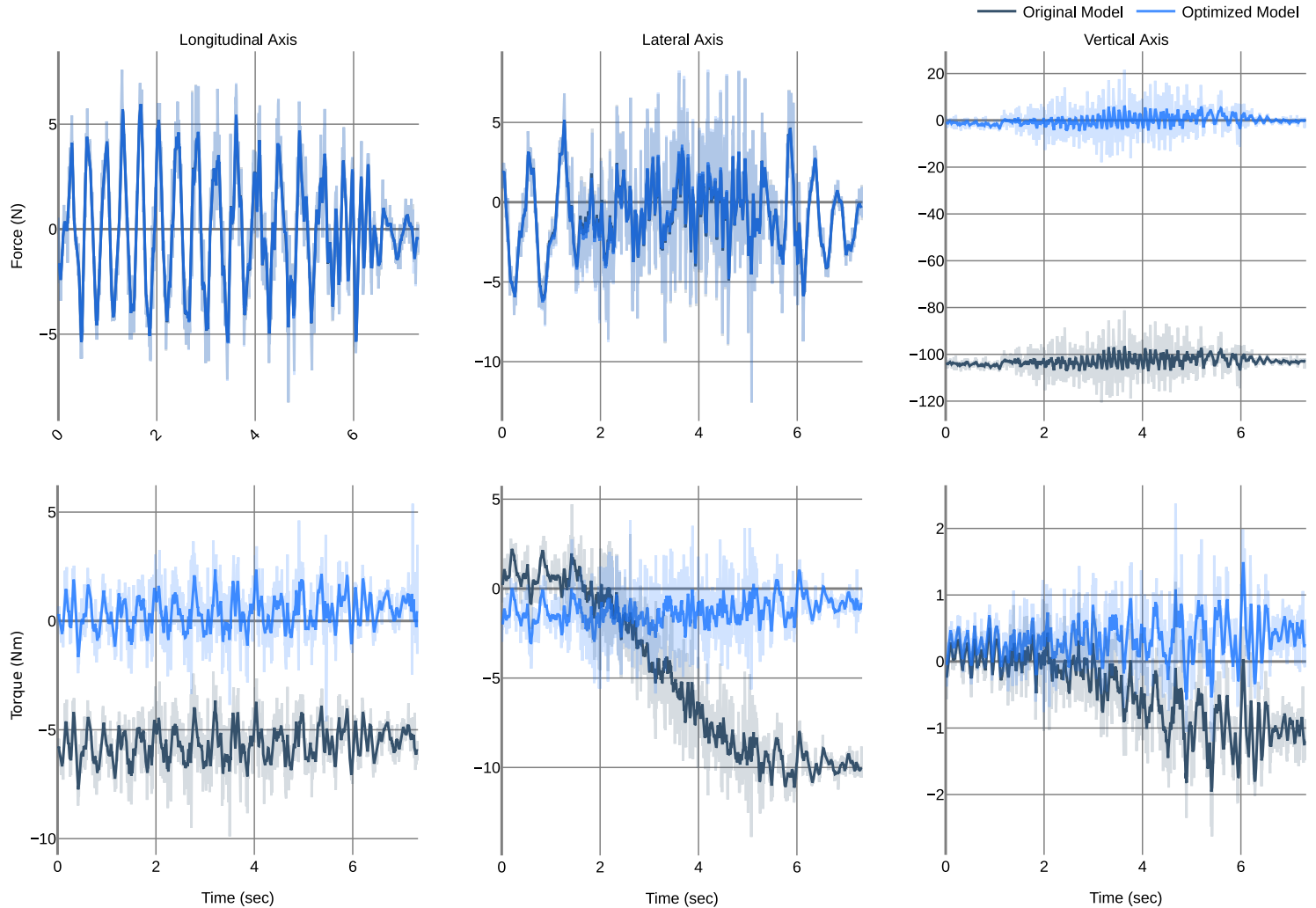

Figure 6. Residual forces (top) and torques (bottom) along all axis of the validating squatmotion. The actual motion takes place between seconds 1 and 6 . We compare the residual forces with the original model (dark-blue) and the optimized model (light-blue) obtained by model identification. We applied a moving mean with a centralized window of 7 frames to reduce the oscillations induced by the COM-stabilizer of the underlying control architecture of the robot. The original data are semi-transparent in the background. 
Table 1. Avg. [std] of the absolute values of residual torques and forces of the base link obtained by inverse dynamics of the squat-motion.

\begin{tabular}{|l|c|c|c|c|c|c|}
\hline Model & $T_{x}[\mathrm{Nm}]$ & $T_{y}[\mathrm{Nm}]$ & $T_{z}[\mathrm{Nm}]$ & $F_{x}[N]$ & $F_{y}[N]$ & $F_{z}[N]$ \\
\hline \hline Original Model & $5.66[0.98]$ & $5.98[4.07]$ & $0.68[0.48]$ & $1.86[1.66]$ & $2.28[1.72]$ & $103.02[4.03]$ \\
\hline Optimized Model & $0.87[0.69]$ & $1.35[1.01]$ & $0.41[0.30]$ & $1.86[1.66]$ & $2.29[1.73]$ & $2.68[3.04]$ \\
\hline
\end{tabular}

of the original data. Therefore, a moving mean with a centralized window of seven frames was applied to the data.

For the acting residual forces about the longitudinal and lateral axis we can not observe any change or improvement (Fig. 6 top). For the acting residual forces about the vertical axis a shift of the force trajectory can be observed, leading to a correction of the absolute values of $F_{z}$ (Tab. 1). The acting residual torques show different signatures about all axis for both models (Fig. 6 bottom). Along the longitudinal axis, a clear offset of the original model of less than $-5 \mathrm{Nm}$ can be observed. This offset is no longer recognizable for the optimized model and an oscillation of almost around zero is shown. For $T_{x}$ the residual torques are smaller for the optimized model (Tab. 1). Along the lateral axis, the original model diverged further into the negative the longer the movement lasted. This trend is no longer visible in the optimized model and for $T_{y}$ smaller residual torques are present (Tab. 1). However, there is a minimal offset to an oscillation around zero again. Similar to $T_{y}$, the residual moments acting around the vertical axis $T_{z}$ diverge further into the negative, even if not to the same extent. This trend was corrected for the optimized model, despite a slightly higher offset to zero than for $T_{y}$. For $T_{z}$ there was only a slight improvement apart from the trend adjustment, as the deviation was already small at the beginning (Tab. 1).

\section{DISCUSSION}

The results show an overall improvement of the model optimized by the model identification procedure. The decrease in distances (Fig. 5), demonstrates that the optimization problem was able to approach a deviation of almost zero by updating the static model parameters. However, this does not represent the quality of the model obtained, but evaluates the process of overall optimization. The fact that the optimization could not minimize all distances to the same extent can be explained by possible over-fitting of certain poses. When generating the poses, as already discussed, all degrees of freedom were systematically covered and supplemented with additional random poses. It appears, however, that some poses are preferred during the optimization. The differences between the individual minimization can possibly be approximated by applying a weighting of the individual poses. Altogether, a minimization in line with expectations was achieved.

The oscillation of residual forces and torques (Fig. 6) is explained by the control architecture of REEM-C which performs COM stabilization in order to prevent a loss of balance. This is particularly evident along the longitudinal and lateral axes, where the robot tries to keep its GCOM close to the center of its support polygon by making minimal compensating movements. For the acting residual forces about the vertical axis a distribution of the unaccounted mass of $\sim 10.5 \mathrm{~kg}$ to the respective segments can be identified, reducing the absolute vertical force $F_{z}$. For the forces acting on the longitudinal and lateral axes, no significant changes are observed. This is due to the fact that the translatory forces acting in the horizontal plane are applied here. These are mainly influenced by the inertial parameters of the segments and the acceleration acting on these segments. Since we have not updated the inertial matrices and the motion is also slow, no change was expected. By analyzing the residual torques along all axes, we can observe two things: the overall quality of the original model and the quality of this mass distribution. The optimization could not only improve the residual torques, but also achieve the improvement while simultaneously incorporating the 
substantial missing masses. The small offset in the residual torques can be explained by a slight over-fitting of the experimental data, minor errors in the marker fitting and small measurement inaccuracies of the force plate and the motion capture system.

\section{CONCLUSION}

Our results show a significant improvement of the model by updating the segment masses and COM locations. We are confident that the mass distributions in this case also correspond to reality. This assumption is partly based on the fact that highly dynamic sit-to-stand movements were possible using this model, which could not be realized with the original model, of course, under the assumption that the inertia matrices already had sufficient quality based on the CAD exports at the beginning. The remaining residual forces and torques are probably also a result of the not yet perfect inertia matrices, although our identification already represents an enormous improvement. This is the next step in this 2-phase approach to iterative model improvement: Based on these values, the inertia parameters can now be estimated on basis of the equation of motion, as in previous work $[12,13,14]$. Of course, for a large number of robots this involves torque estimation, errorprone data due to finite differences, or noisy data due to possible control architecture. However, a very accurate approximation of the static parameters of the dynamic model has already been realized. The absence of possible redundancies in the optimization problem due to simultaneous determination of all dynamic parameters is also ensured. The further improvement of the model and also the use of the proposed model and its further analysis on the basis of highly dynamic sit-to-stand transitions will be part of a future publication.

\section{ACKNOWLEDGMENTS}

This work was supported by the project EUROBENCH funded by H2020 Topic ICT 27-2017 under grantagreement number 779963. We thank PAL Robotics for the opportunity to work with the REEM-C robot within the EUROBENCH project.

\section{REFERENCES}

[1] Kheddar, A., Caron, S., Gergondet, P., Comport, A., Tanguy, A., Ott, C., Henze, B., Mesesan, G., Englsberger, J., Roa, M.A., et al.: Humanoid robots in aircraft manufacturing: The airbus use cases. IEEE Robotics \& Automation Magazine 26(4) (2019) 30-45

[2] Jung, T., Lim, J., Bae, H., Lee, K.K., Joe, H.M., Oh, J.H.: Development of the humanoid disaster response platform DRC-HUBO+. IEEE Transactions on Robotics 34(1) (2018) 1-17

[3] Asfour, T., Waechter, M., Kaul, L., Rader, S., Weiner, P., Ottenhaus, S., Grimm, R., Zhou, Y., Grotz, M., Paus, F.: ARMAR-6: A high-performance humanoid for human-robot collaboration in real-world scenarios. IEEE Robotics \& Automation Magazine 26(4) (2019) 108-121

[4] Al-Shuka, H.F., Allmendinger, F., Corves, B., Zhu, W.H.: Modeling, stability and walking pattern generators of biped robots: a review. Robotica 32(6) (2014) 907-934

[5] Goswami, A.: Postural stability of biped robots and the foot-rotation indicator (FRI) point. The International Journal of Robotics Research 18(6) (1999) 523-533

[6] Griffin, R.J., Wiedebach, G., Bertrand, S., Leonessa, A., Pratt, J.: Walking stabilization using step timing and location adjustment on the humanoid robot, atlas. In: 2017 IEEE/RSJ International Conference on Intelligent Robots and Systems (IROS), IEEE (2017) 667-673

[7] Kashiri, N., Abate, A., Abram, S.J., Albu-Schaffer, A., Clary, P.J., Daley, M., Faraji, S., Furnemont, R., Garabini, M., Geyer, H., et al.: An overview on principles for energy efficient robot locomotion. Frontiers in Robotics and AI 5 (2018) 129 
[8] Atkeson, C.G., An, C.H., Hollerbach, J.M.: Estimation of inertial parameters of manipulator loads and links. The International Journal of Robotics Research 5(3) (1986) 101-119

[9] Armstrong, B., Khatib, O., Burdick, J.: The explicit dynamic model and inertial parameters of the PUMA $560 \mathrm{arm}$. In: Proceedings. 1986 IEEE international conference on robotics and automation. Volume 3., IEEE (1986) 510-518

[10] Sujan, V.A., Dubowsky, S.: An optimal information method for mobile manipulator dynamic parameter identification. IEEE/ASME transactions on mechatronics 8(2) (2003) 215-225

[11] Venture, G., Ayusawa, K., Nakamura, Y.: Motion capture based identification of the human body inertial parameters. In: 2008 30th Annual International Conference of the IEEE Engineering in Medicine and Biology Society, IEEE (2008) 4575-4578

[12] Ayusawa, K., Venture, G., Nakamura, Y.: Identification of the inertial parameters of a humanoid robot using unactuated dynamics of the base link. In: Humanoids 2008-8th IEEERAS International Conference on Humanoid Robots, IEEE (2008) 1-7

[13] Ayusawa, K., Venture, G., Nakamura, Y.: Identification of humanoid robots dynamics using floating-base motion dynamics. In: 2008 IEEE/RSJ International Conference on Intelligent Robots and Systems, IEEE (2008) 2854-2859

[14] Mistry, M., Schaal, S., Yamane, K.: Inertial parameter estimation of floating base humanoid systems using partial force sensing. In: 2009 9th IEEE-RAS International Conference on Humanoid Robots, IEEE (2009) 492-497

[15] Gautier, M., Khalil, W.: Exciting trajectories for the identification of base inertial parameters of robots. The International journal of robotics research 11(4) (1992) 362-375

[16] Swevers, J., Ganseman, C., Tukel, D.B., De Schutter, J., Van Brussel, H.: Optimal robot excitation and identification. IEEE transactions on robotics and automation 13(5) (1997) 730-740

[17] Ogawa, Y., Venture, G., Ott, C.: Dynamic parameters identification of a humanoid robot using joint torque sensors and/or contact forces. In: 2014 IEEE-RAS International Conference on Humanoid Robots, IEEE (2014) 457-462

[18] Aller, F., Harant, M., Sontag, S., Millard, M., Mombaur, K.: I3SA: The Increased Step Size Stability Assessment Benchmark and its Application to the Humanoid Robot REEMC. In: 2021 IEEE/RSJ International Conference on Intelligent Robots and Systems (IROS), submitted and accepted, IEEE (2021)

[19] Koch, K.H., Mombaur, K., Stasse, O., Soueres, P.: Optimization based exploitation of the ankle elasticity of HRP-2 for overstepping large obstacles. In: 2014 IEEE-RAS International Conference on Humanoid Robots, IEEE (2014) 733-740

[20] Marquard, D.W.: An Algorithm for Least-Squares Estimation of Nonlinear Parameters. Journal of the Society for Industrial and Applied Mathematics 11(2) (1963) 431-441

[21] Felis, M.L.: RBDL: an efficient rigid-body dynamics library using recursive algorithms. Autonomous Robots 41(2) (2017) 495-511

[22] Leineweber, D., Schäfer, A., Bock, H., Schlöder, J.: An efficient multiple shooting based reduced SQP strategy for large-scale dynamic process optimization: Part II: Software aspects and applications. Computers \& Chemical Engineering 27(2) (2003) 167-174 